\title{
Existence and asymptotic behavior results of positive periodic solutions for discrete-time logistic model
}

Bo Du' ${ }^{1,2}$ and Shouli Zhu ${ }^{1 *}$

\section{"Correspondence:}

zhusl2010@126.com

${ }^{1}$ Department of Mathematics,

Huaiyin Normal University, Huaian,

Jiangsu 223300, China

Full list of author information is

available at the end of the article

\begin{abstract}
A discrete-time logistic model with delay is studied. The existence of a positive periodic solution for a discrete-time logistic model is obtained by a continuation theorem of coincidence degree theory, and a sufficient condition is given to guarantee the global exponential stability of a periodic solution. Finally, an example is given to show the effectiveness of the results in this paper.
\end{abstract}

Keywords: discrete; delay; periodic solution; stability

\section{Introduction}

Examples of the discrete phenomena in nature abound and somehow the continuous version have commandeered all our attention - perhaps owing to that special mechanism in human nature that permits us to notice only what we have been conditioned to. The theory of difference equations has grown at an accelerated rate in the past decades; see [1-3].

In this paper, we discuss the existence and exponential stability of positive periodic solution for the following logistic model:

$$
\left\{\begin{array}{l}
x(n+1)=\frac{\alpha(n) x(n)}{1+\beta(n) x(n-\tau)}, \quad n \in Z, \\
x(n)=\phi(n)>0, \quad n \in[-\tau, 0]_{Z},
\end{array}\right.
$$

where $\alpha(n)>1, \beta(n)>0$ are $N$-periodic sequences, $\tau$ is a positive integer,

$$
[a, b]_{Z}=\{a, a+1, \ldots, b-1, b\} \quad \text { for } a, b \in Z \text { and } a \leq b .
$$

There have been many papers concerned with the properties of solution of difference equations. In [4], Zhang et al. studied the existence of periodic solutions of the equation without delay

$$
x(n+1)=\mu x(n)[1-x(n) / k]+b(n), \quad n \in Z,
$$

under the assumptions that $\mu \in(1,2),|b(n)|<\frac{(\mu-1)^{2}}{4 \mu} k$ hold for all $n \in Z$. Parhi [5] considered the delay difference equation

$$
y(n+1)-y(n)+q_{n} G(y(n-k))=b(n), \quad n \in Z,
$$

(c) 2015 Du and Zhu. This article is distributed under the terms of the Creative Commons Attribution 4.0 International License (http://creativecommons.org/licenses/by/4.0/), which permits unrestricted use, distribution, and reproduction in any medium, provided you give appropriate credit to the original author(s) and the source, provide a link to the Creative Commons license, and indicate if changes were made. 
and obtained the oscillatory and asymptotic behavior of solutions of (1.2). Liu and Ge [6] considered the difference equation

$$
y(n+1)-y(n)=p_{n} f(y(n-k))+r(n), \quad n \in Z,
$$

where $p(n)$ is nonnegative, $r(n)$ is a real numbers sequence. Suppose $\lim _{x \rightarrow 0} \frac{f(x)}{x}=-b<0$ and $x f(x)<0,|f(x)| \leq \gamma|x|$ for all $x \neq 0$, the authors proved that if

$$
\mu=\gamma \sum_{s=n-k}^{s} p_{s}<1.5+\frac{1}{2(k+1)}, \quad \sum_{s=1}^{+\infty} p_{s}=+\infty, \quad \lim _{n \rightarrow+\infty} \frac{r_{n}}{p_{n}}=0
$$

then every solution of (1.3) tends to zero as $\mathrm{n}$ tends to infinity. Li et al. [7] used the upper and lower solutions method to show that there exists a $\lambda^{*}>0$ such that the nonlinear functional difference equation

$$
x(n+1)=a(n) x(n)+\lambda(n) x(n)+\lambda h(n) f(x(n-\tau(n))), \quad n \in Z,
$$

has at least one positive $T$-periodic solutions for $\lambda \in\left(0, \lambda^{*}\right)$ and does not have any positive $T$-periodic solutions for $\lambda>\lambda^{*}$, where $a(n), h(n)$, and $\tau(n)$ are $T$-periodic solutions. Jiang et al. [8] presented the optimal existence theory for single and multiple positive periodic solutions to a class of functional difference equations based upon the fixed point theorem in cones.

For a complicated dynamical system, we note that discrete-time neural networks have been studied by many authors; see e.g. Hu and Wang [9], Wang and Xu [10], Xiong and Cao [11], Yuan et al. [12], Zhao and Wang [13] and Zou and Zhou [14] for DNNs without time delays and Chen et al. [15], Liang et al. [16], Liang et al. [17] and Xiang et al. [18] for DNNs with discrete time delays. For more related results, see [19-28].

So far, to the best of the authors knowledge, there are few results for the existence and stability of positive periodic solutions to (1.1). The major challenges are as follows: (1) In order to obtain existence of positive periodic solutions, we must change (1.1) to the proper form by a variable transformation. How can we choose the above variable transformation, which is the key to the study of (1.1)? (2) Since it is very difficult to construct a Lypunov functional to (1.1), how can we choose a proper special function for obtaining the stability results, which is significant to our proof? (3) It is non-trivial to establish a unified framework.

It is, therefore, the main purpose of this paper to make the first attempt to handle the listed challenges.

Remark 1.1 Equation (1.1) was proposed by Pielou [29] in 1974, which is a discrete analog of the delay logistic equation

$$
x^{\prime}(t)=r(t) x(t)\left(1-\frac{x(t-\tau)}{p}\right) .
$$

A classic logistic model has received great attention from theoretical and mathematical biologists and has been well studied; see e.g. [29-32]. 
The following sections are organized as follows: In Section 2, the existence of positive periodic solution to (1.1) is obtained. In Section 3, sufficient conditions are established for the global exponential stability of (1.1). In Section 4, an example is given to show the feasibility of our results.

\section{Existence of positive periodic solution}

Let $X$ and $Y$ be real Banach spaces and let $L: D(L) \subset X \rightarrow Y$ be a Fredholm operator with index zero, here $D(L)$ denotes the domain of $L$. This means that $\operatorname{Im} L$ is closed in $Y$ and $\operatorname{dim} \operatorname{Ker} L=\operatorname{codim} \operatorname{Im} L<+\infty$. If $L$ is a Fredholm operator with index zero, then there exist continuous projectors $P: X \rightarrow X, Q: Y \rightarrow Y$ such that $\operatorname{Im} P=\operatorname{Ker} L, \operatorname{Im} L=\operatorname{Ker} Q=$ $\operatorname{Im}(I-Q)$. It follows that $L_{D(L) \cap \operatorname{Ker} P}:(I-P) X \rightarrow \operatorname{Im} L$ is invertible. Denote by $K_{p}$ the inverse of $L_{P}$.

Let $\Omega$ be an open bounded subset of $X$, a map $N: \bar{\Omega} \rightarrow Y$ is said to be $L$-compact in $\bar{\Omega}$ if $Q N(\bar{\Omega})$ is bounded and the operator $K_{p}(I-Q) N(\bar{\Omega})$ is relatively compact. Because $\operatorname{Im} Q$ is isomorphic to $\operatorname{Ker} L$, there exists an isomorphism $J: \operatorname{Im} Q \rightarrow \operatorname{Ker} L$. We first recall the famous Mawhin continuation theorem.

Lemma 2.1 [33] Suppose that $X$ and $Y$ are two Banach spaces, and $L: D(L) \subset X \rightarrow Y$, is a Fredholm operator with index zero. Furthermore, $\Omega \subset X$ is an open bounded set and $N: \bar{\Omega} \rightarrow Y$ is L-compact on $\bar{\Omega}$. If all the following conditions hold:

(1) $L x \neq \lambda N x, \forall x \in \partial \Omega \cap D(L), \forall \lambda \in(0,1)$,

(2) $N x \notin \operatorname{Im} L, \forall x \in \partial \Omega \cap \operatorname{Ker} L$,

(3) $\operatorname{deg}\{J Q N, \Omega \cap \operatorname{Ker} L, 0\} \neq 0$,

where $J: \operatorname{Im} Q \rightarrow \operatorname{Ker} L$ is an isomorphism, then the equation $L x=N x$ has a solution on $\bar{\Omega} \cap D(L)$.

Lemma 2.2 [34] Let $g: Z \rightarrow R$ be $\omega$-periodic. Then for any fixed $k_{1}, k_{2} \in I_{\omega}$, and any $k \in Z$, one has

$$
g(k) \leq g\left(k_{1}\right)+\sum_{s=0}^{\omega-1}|g(s+1)-g(s)|
$$

and

$$
g(k) \geq g\left(k_{2}\right)-\sum_{s=0}^{\omega-1}|g(s+1)-g(s)|
$$

Now, we state the main results and give its proof.

Theorem 2.1 Suppose that assumptions $\left(\mathrm{H}_{1}\right)$ and $\left(\mathrm{H}_{2}\right)$ hold:

$\left(\mathrm{H}_{1}\right)$ there exists a constant $C>0$ such that if $x(n)$ is a $N$-periodic sequences and satisfies

$$
\sum_{n=0}^{N-1}\left[-\ln \left(1+\beta(n) e^{y(n-\tau)}\right)+\ln \alpha(n)\right]=0
$$

then we have

$$
\sum_{n=0}^{N-1}\left|\ln \left(1+\beta(n) e^{y(n-\tau)}\right)+\ln \alpha(n)\right| \leq C ;
$$


$\left(\mathrm{H}_{2}\right)$ there exists a constant $D>0$ such that when $y>D$,

$$
\ln \left(1+\beta(n) e^{y(n)}\right)>\ln \alpha(n)
$$

and

$$
\ln \left(1+\beta(n) e^{-y(n)}\right)<\ln \alpha(n)
$$

uniformly hold for $n \in Z$.

Then (1.1) has at least one positive $N$-periodic solution.

Proof In order to obtain the positive periodic solution of (1.1), let $x(n)=e^{y(n)}$, then from (1.1) we have

$$
y(n+1)-y(n)=-\ln \left(1+\beta(n) e^{y(n-\tau)}\right)+\ln \alpha(n), \quad n \in Z,
$$

with the initial condition

$$
y(n)=\ln \phi(n):=\psi(n), \quad n \in[-\tau, 0]_{Z},
$$

where $\alpha(n)>1, \beta(n)>0$ are $N$-periodic sequences, $\tau$ is a positive integer. Define

$$
l=\{x=\{x(n)\}, x(n) \in R, n \in Z\} .
$$

Let $l_{N} \subset l$ denote the subspace of all $N$-periodic sequences equipped with the norm

$$
\|x\|=\max _{n \in I_{N}}|x(n)| \quad \text { for any } x \in l_{N}
$$

where $I_{N}=\{0,1,2, \ldots, N-1\}$. Then $l_{N}$ is a Banach space. Let

$$
l_{N}^{0}=\left\{y(n) \in l_{N}: \sum_{n=0}^{N-1} y(n)=0\right\}, \quad l_{N}^{c}=\left\{y(n) \in l_{N}: y(n)=\text { constant }, n \in Z\right\} .
$$

Then $l_{N}^{0}$ and $l_{N}^{c}$ are both closed linear subspaces of $l_{N}$, and $l_{N}=l_{N}^{0} \oplus l_{N}^{c}$, $\operatorname{dim} l_{N}^{c}=1$. Take $X=Y=l_{N}$. Now, for $y \in X, n \in Z$, we define a linear operator

$$
(L y)(n)=y(n+1)-y(n)
$$

and a nonlinear operator

$$
(N y)(n)=-\ln \left(1+\beta(n) e^{y(n-\tau)}\right)+\ln \alpha(n) .
$$

Then $L$ is a bounded linear operator with $\operatorname{Ker} L=l_{N}^{c}$ and $\operatorname{Im} L=l_{N}^{0}$. So it follows that $L$ is a Fredholm mapping of index zero. Define the continuous projectors $P, Q$,

$$
P: X \rightarrow \operatorname{Ker} L, \quad(P x)(n)=\frac{1}{N} \sum_{n=0}^{N-1} x(n)
$$


and

$$
Q: Y \rightarrow Y / \operatorname{Im} L, \quad Q y=\frac{1}{N} \sum_{n=0}^{N-1} y(n) .
$$

Let

$$
L_{P}=\left.L\right|_{D(L) \cap \operatorname{Ker} P}: D(L) \cap \operatorname{Ker} P \rightarrow \operatorname{Im} L,
$$

then

$$
L_{P}^{-1}=K_{p}: \operatorname{Im} L \rightarrow D(L) \cap \operatorname{Ker} P .
$$

Since $\operatorname{Im} L \subset Y$ and $D(L) \cap \operatorname{Ker} P \subset X, K_{p}$ is an embedding operator. Hence $K_{p}$ is a completely operator in $\operatorname{Im} L$. By the definitions of $Q$ and $N$, one knows that $Q N(\bar{\Omega})$ is bounded on $\bar{\Omega}$. Hence the nonlinear operator $N$ is $L$-compact on $\bar{\Omega}$. We complete the proof in three steps.

Step 1. Let $\Omega_{1}=\{y \in D(L) \subset X: L y=\lambda N y, \lambda \in(0,1)\}$. We show that $\Omega_{1}$ is a bounded set. If $\forall y \in \Omega_{1}$, then $L y=\lambda N y$, i.e.,

$$
y(n+1)-y(n)=\lambda\left[-\ln \left(1+\beta(n) e^{y(n-\tau)}\right)+\ln \alpha(n)\right]
$$

$\forall x \in \Omega_{1}$, summing on both sides of (2.2) from 0 to $N-1$ with respect to $n$, we have

$$
\sum_{n=0}^{N-1}\left[-\ln \left(1+\beta(n) e^{y(n-\tau)}\right)+\ln \alpha(n)\right]=0 .
$$

Thus, from (2.3) and condition $\left(\mathrm{H}_{1}\right)$ we obtain

$$
\sum_{n=0}^{N-1}|y(n+1)-y(n)| \leq \sum_{n=0}^{N-1}\left|-\ln \left(1+\beta(n) e^{y(n-\tau)}\right)+\ln \alpha(n)\right| \leq C
$$

We claim that there exist a point $k \in Z$ and a constant $M_{1}>0$ such that

$$
y(k-\tau)<M_{1} .
$$

Otherwise, for any $M_{1}>0$ and each $n \in I_{N}$, one has

$$
y(n-\tau) \geq M_{1}
$$

In view of assumption $\left(\mathrm{H}_{2}\right)$, we see that this contradicts (2.3). Hence (2.4) holds. Denote

$$
k-\tau=\xi_{1}+p N, \quad \xi_{1} \in I_{N}, p \in Z .
$$

Then

$$
y\left(\xi_{1}\right)<M_{1} \text {. }
$$


In a similar way, from (2.3) and assumption $\left(\mathrm{H}_{2}\right)$, there exist a point $\xi_{2} \in I_{N}$ and a constant $M_{2}>0$ such that

$$
y\left(\xi_{2}\right)>-M_{2}
$$

Therefore, it follows from Lemma 2.2, (2.1), (2.5), and (2.6) that

$$
y(n) \leq y\left(\xi_{1}\right)+\sum_{n=0}^{N-1}|y(n+1)-y(n)|<M_{1}+C
$$

and

$$
y(n) \geq y\left(\xi_{2}\right)-\sum_{n=0}^{N-1}|y(n+1)-y(n)|>-M_{2}-C .
$$

Thus

$$
\|y\|<\max \left\{M_{1}+C, M_{2}+C\right\}:=M \text {. }
$$

Step 2. We will show that condition (2) in Lemma 2.1 satisfies. Let

$$
\Omega=\left\{y \in X \mid \max _{n \in I_{N}}\|y\|<A\right\},
$$

where $A=\max \{M, D\}$. Obviously, condition (1) in Lemma 2.1 satisfies. When $\forall y \in \partial \Omega \cap$ $\operatorname{Ker} L, y$ is a constant with $\|y\|=A$. Then we claim $Q N y \neq 0$. In fact, if $Q N y=0$, then

$$
\sum_{n=0}^{N-1}\left[-\ln \left(1+\beta(n) e^{y(n-\tau)}\right)+\ln \alpha(n)\right]=0,
$$

which contradicts assumption $\left(\mathrm{H}_{2}\right)$ when $\|y\|=A$.

Step 3. We will show that condition (3) in Lemma 2.1 holds. Take the homotopy

$$
H(y, \mu)=\mu y+(1-\mu) Q N y, \quad y \in \bar{\Omega} \cap \operatorname{Ker} L, \mu \in[0,1] .
$$

We claim $H(y, \mu) \neq 0$ for all $y \in \partial \Omega \cap \operatorname{Ker} L$. If this is not true, then

$$
-\mu y=\frac{1-\mu}{N} \sum_{n=0}^{N-1}\left[\ln \left(1+\beta(n) e^{y(n-\tau)}\right)+\ln \alpha(n)\right] .
$$

Since $y \in \partial \Omega \cap \operatorname{Ker} L, \mu \in[0,1], y H(y, \mu)>0$, one has $H(y, \mu) \neq 0$. By the degree theory,

$$
\begin{aligned}
\operatorname{deg}\{Q N, \Omega \cap \operatorname{Ker} L, 0\} & =\operatorname{deg}\{H(\cdot, 0), \Omega \cap \operatorname{Ker} L, 0\} \\
& =\operatorname{deg}\{H(\cdot, 1), \Omega \cap \operatorname{Ker} L, 0\} \\
& =\operatorname{deg}\{I, \Omega \cap \operatorname{Ker} L, 0\} \neq 0 .
\end{aligned}
$$

From Lemma 2.1, we know that $L x(n)=N x(n)$ has at least one periodic solution in $\bar{\Omega}$. That is, (1.1) has at least one positive $N$-periodic solution. The proof is completed. 
Corollary 2.1 Let $\mathcal{F}(n, y)=-\ln \left(1+\beta(n) e^{y(n-\tau)}\right)+\ln \alpha(n), n \in Z, y \in R$. There exist constants $D_{1}$ and $D_{2}$ such that

(i) $y \mathcal{F}(n, y)>0$ for $|y|>D_{1}, n \in I_{N}$,

(ii) one of the following two conditions holds:

(a) $y \mathcal{F}(n, y) \leq D_{2}$ for $y \geq D_{1}, n \in I_{N}$,

(b) $y \mathcal{F}(n, y) \geq D_{2}$ for $y \leq-D_{1}, n \in I_{N}$.

Then (2.2) has at least one periodic solution.

Remark 2.1 The initial condition $x(n)=\phi(n)>0, n \in[-\tau, 0]_{Z}$, of (1.1) assures that the initial condition $y(n)=\ln x(n),, n \in[-\tau, 0]_{Z}$, of (2.1) is meaningful.

Remark 2.2 In [35], Li and Huo studied a class of abstract delay difference equation and obtained the existence of positive periodic solutions. In the present paper, based on the work of [35], we investigate a concrete model and obtain the population dynamics of the model.

\section{Global exponential stability of periodic solution}

In this section, we establish some results for exponential stability of the $N$-periodic solution of (1.1).

Definition 3.1 The periodic solution of $(2.1), y^{*}(n)$ is globally exponentially stable if there exist constants $\mu>1$ and $L>0$ such that

$$
\left|y(n)-y^{*}(n)\right| \leq L\left\|\psi-\psi^{*}\right\| \mu^{-n}, \quad n \in Z^{+},
$$

where $y(n)$ is a solution of (1.1) with the initial value condition $y(n)=\psi(n), \psi^{*}$ is the initial value of $y^{*}(n)$, and

$$
\left\|\psi-\psi^{*}\right\|=\max _{n \in[-\tau, 0] Z}\left|\psi(n)-\psi^{*}(n)\right| .
$$

Theorem 3.1 Under the conditions of Theorem 2.1, assume further that:

(i)

$$
\alpha^{+}+\lambda_{0}^{\tau+1} L \beta^{+}<1
$$

where $\alpha^{+}=\max \{\alpha(n), n \in Z\}, \beta^{+}=\max \{\beta(n), n \in Z\}, \lambda_{0}>1$ with

$$
\lambda_{0}\left(\alpha^{+}+\lambda_{0}^{\tau+1} L \beta^{+}\right)<1
$$

(ii) If $f(x, y)=x y, x, y \in R$, then $\left|f(x, y)-f\left(x^{*}, y *\right)\right| \leq L\left|y-y^{*}\right|$, where $L$ is a positive constant.

Then system (1.1) has a $N$-periodic solution $x^{*}(n)$, and there exists $\lambda_{0}>1$ such that

$$
\left|x(n)-x^{*}(n)\right| \leq \lambda_{0}^{-n} \max _{s \in[-\tau, 0] Z}\left|\psi(s)-\psi^{*}(s)\right| .
$$

Proof By (1.1), we have

$$
x(n+1)-x^{*}(n+1)=\alpha(n)\left[x(n)-x^{*}(n)\right]+\beta(n)\left[x^{*}(n+1) x^{*}(n-\tau)-x(n+1) x(n-\tau)\right] .
$$


For $\lambda \in R$, define the function

$$
F(\lambda)=1-\lambda+\lambda\left[1-\alpha^{+}-\lambda_{0}^{\tau+1} L \beta^{+}\right]
$$

From condition (i), we have $F(1)>0$. So, there exists some constant $\lambda_{0}>1$ such that $F\left(\lambda_{0}\right)>0$. Then by (3.1), we have

$$
\left|x(n+1)-x^{*}(n+1)\right| \leq \alpha^{+}\left|x(n)-x^{*}(n)\right|+\beta^{+}\left|x^{*}(n+1) x^{*}(n-\tau)-x(n+1) x(n-\tau)\right| .
$$

Define $u(n)=\lambda_{0}^{n}\left|x(n)-x^{*}(n)\right|, n \in[-\tau,+\infty)_{Z}$, then by (3.2) and condition (ii), we have

$$
u(n+1) \leq \lambda_{0} \alpha^{+} u(n)+\lambda_{0}^{\tau+1} L \beta^{+} u(n-\tau)
$$

Assume that $K=\max _{s \in[-\tau, 0]_{Z}}\left|\psi(s)-\psi^{*}(s)\right|$. Then we claim that

$$
u(n) \leq K, \quad n \in Z^{+} .
$$

Otherwise, there exists $n_{0} \in Z^{+}$such that

$$
u(n) \leq K, \quad n \in\left[-\tau, n_{0}-1\right]_{Z}, \quad u\left(n_{0}\right)>K .
$$

By (3.3) and condition (i) we have

$$
\begin{aligned}
K & <u\left(n_{0}\right) \leq \lambda_{0} \alpha^{+} u\left(n_{0}-1\right)+\lambda_{0}^{\tau+1} L \beta^{+} u\left(n_{0}-1-\tau\right) \\
& \leq \lambda_{0} \alpha^{+} K+\lambda_{0}^{\tau+1} L \beta^{+} K \\
& =\lambda_{0}\left(\alpha^{+}+\lambda_{0}^{\tau+1} L \beta^{+}\right) K<K,
\end{aligned}
$$

which is a contradiction. So $u(n) \leq K, n \in Z^{+}$. Therefore,

$$
\left|x(n)-x^{*}(n)\right| \leq \lambda_{0}^{-n} \max _{s \in[-\tau, 0]_{Z}}\left|\psi(s)-\psi^{*}(s)\right|
$$

The proof is completed.

Remark 3.1 Because (3.2) contains the nonlinear term $x^{*}(n+1) x^{*}(n-\tau)-x(n+1) x(n-\tau)$, which results in great difficulty in obtaining exponential stability, we add condition (ii).

Remark 3.2 In general, the Lyapunov functional method is crucial for studying stability problems. In the present paper, due to the stronger non-linearity of (1.1), the Lyapunov functional method is not valid. We overcome these difficulties by constructing a novel functional, which is different for the corresponding ones of past work.

\section{Numerical simulations}

This section presents an example to demonstrate the validity of our theoretical results:

$$
x(n+1)=\frac{\frac{5-\cos n \pi}{3} x(n)}{1+0.2 x(n-\tau)}, \quad n \in Z
$$



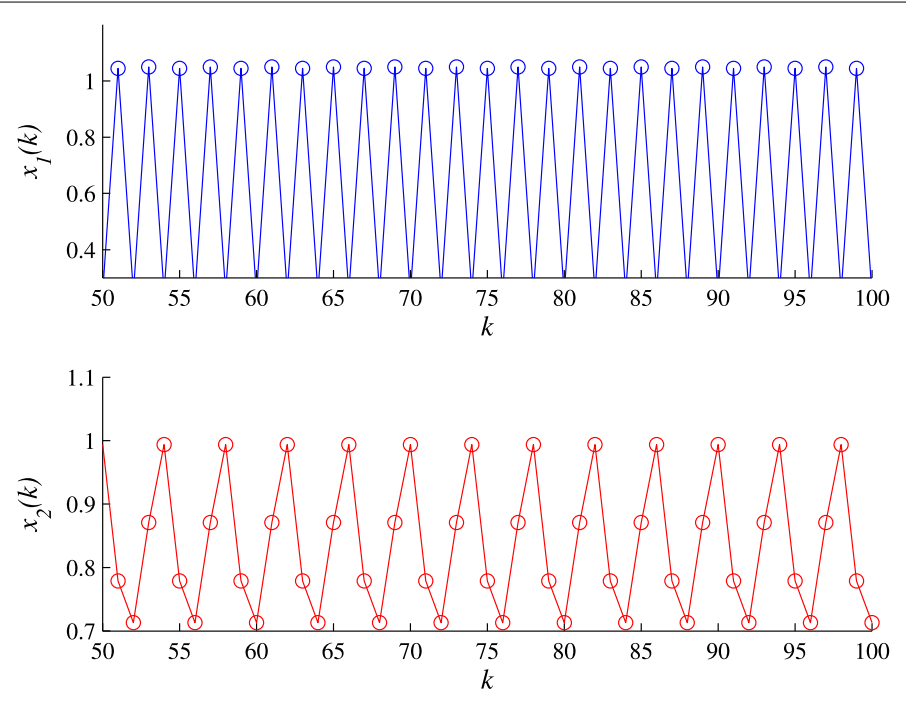

Figure 1 Evolution of the $x(k)$ of $(4.1)$ with $k$ from 50 to 100.

where $\alpha(n)=\frac{5-\cos n \pi}{3}>1, \beta(n)=0.2$. We can choose a proper parameter $\tau$ and $L$ such that all conditions of Theorem 3.1 hold. So there exists a periodic solution for (4.1) which is globally exponentially stable. The corresponding numerical simulations are presented in Figure 1 with different initial conditions.

In this paper, we discussed the existence and stability of positive periodic solutions for (1.1). First, the sufficient conditions that ensure the existence of a positive periodic solution were obtained by using the continuation theorem and some inequality techniques. Then a non-Lyapunov method was used to establish the criteria for the global exponential stability of the periodic solution. Finally, a numerical example was presented to demonstrate the effectiveness of our theoretical results. The proposed criteria in this paper are easy to verify. The proposed analysis method is also easy to extend to the case of other differential equations.

\section{Competing interests}

The authors declare that they have no competing interests.

Authors' contributions

All authors contributed equally to the writing of this paper. All authors read and approved the final manuscript.

\section{Author details}

'Department of Mathematics, Huaiyin Normal University, Huaian, Jiangsu 223300, China. ${ }^{2}$ Department of Mathematics, Yangzhou University, Yangzhou, Jiangsu 225002, China.

\section{Acknowledgements}

This paper is supported by the Postdoctoral Foundation of Jiangsu (1402113C) and the Postdoctoral Foundation of China (2014M561716).

Received: 2 February 2015 Accepted: 3 June 2015 Published online: 17 June 2015

\section{References}

1. Agarwal, RP, O'Regan, D, Wong, PJY: Positive Solutions of Differential, Difference and Integral Equations. Kluwer Academic, Dordrecht (1999)

2. Agarwal, RP: Difference Equations and Inequalities: Theory, Methods, and Applications, 2nd edn. Monographs and Textbooks in Pure and Applied Mathematics, vol. 228. Dekker, New York (2000)

3. Agarwal, RP, O'Regan, D: Infinite Interval Problems for Differential, Difference and Integral Equations. Kluwer Academic, Dordrecht (2001)

4. Zhang, R, Wang, Z, Chen, Y: Periodic solutions of a single species discrete population model with periodic harvest/stock. Comput. Math. Appl. 39, 77-90 (2000) 
5. Parhi, N: Behavior of solutions of delay differential equations of first order. Indian J. Pure Appl. Math. 33, 31-34 (2002)

6. Liu, Y: Ge, W, Global asymptotic behavior of a forced difference equation. Comput. Math. Appl. 47, 1177-1193 (2004)

7. Li, Y, Zhu, L, Liu, P: Positive periodic solutions of nonlinear functional difference equations depending on a parameter. Comput. Math. Appl. 48, 1453-1459 (2004)

8. Jiang, D, O'Regan, D, Agarwal, RP: Optimal existence theory for single and multiple positive periodic solutions to functional difference equations. Appl. Math. Lett. 161, 441-462 (2005)

9. Hu, S, Wang, J: Global robust stability of a class of discrete-time interval neural networks. IEEE Trans. Circuits Syst. I, Regul. Pap. 53, 129-138 (2006)

10. Wang, L, Xu, Z: Sufficient and necessary conditions for global exponential stability of discrete-time recurrent neural networks. IEEE Trans. Circuits Syst. I, Regul. Pap. 53, 1373-1380 (2006)

11. Xiong, W, Cao, J: Global exponential stability of discrete-time Cohen-Grossberg neural networks. Neurocomputing $64,433-446(2005)$

12. Yuan, Z, Hu, D, Huang, L: Stability and bifurcation analysis on a discrete-time neural network. J. Comput. Appl. Math. $177,89-100(2005)$

13. Zhao, H, Wang, L: Stability and bifurcation for discrete-time Cohen-Grossberg neural network. Appl. Math. Comput. $179,787-798$ (2006)

14. Zou, L, Zhou, Z: Periodic solutions for nonautonomous discrete-time neural networks. Appl. Math. Lett. 19, 174-185 (2006)

15. Chen, W, Lu, X, Liang, D: Global exponential stability for discrete-time neural networks with variable delays. Phys. Lett. A 358, 186-198 (2006)

16. Liang, J, Cao, J, Lam, J: Convergence of discrete-time recurrent neural networks with variable delay. Int. J. Bifurc. Chaos 15, 581-595 (2005)

17. Liang, J, Cao, J, Ho, D: Discrete-time bidirectional associative memory neural networks with variable delays. Phys. Lett. A 335, 226-234 (2005)

18. Xiang, H, Yan, K, Wang, B: Existence and global stability of periodic solution for delayed discrete high-order Hopfield-type neural networks. Discrete Dyn. Nat. Soc. 3, 281-297 (2005)

19. Hu, Z, Tang, Z, Jiang, H: Stability analysis in a class of discrete SIRS epidemic models. Nonlinear Anal., Real World Appl. $13,2017-2033(2012)$

20. Zhang, R, Chen, Z: Value distribution of difference polynomials of meromorphic functions. Sci. Sin., Math. 42, $1115-1130$ (2012)

21. Latreuch, Z, Belaidi, B: Growth and oscillation of meromorphic solutions of linear difference equations. Mat. Vesn. 66, 213-222 (2014)

22. Zheng, X, Tu, J: Growth of meromorphic solutions of linear difference equations. J. Math. Anal. Appl. 384, 349-356 (2011)

23. Chen, ZX: Zeros of entire solutions to complex linear difference equations. Acta Math. Sci. Ser. B Engl. Ed. 32 1141-1148 (2012)

24. Gao, C: Solutions to discrete multiparameter periodic boundary value problems involving the $p$-Laplacian via critical point theory. Acta Math. Sci. Ser. B Engl. Ed. 34, 1225-1236 (2014)

25. Henderson, J, Luca, R: Existence of positive solutions for a system of second-order multi-point discrete boundary value problems. J. Differ. Equ. Appl. 19, 1889-1906 (2013)

26. Henderson, J, Luca, R: On a second-order nonlinear discrete multi-point eigenvalue problem. J. Differ. Equ. Appl. 20, 1005-1018 (2014)

27. Yuan, $C$, Wen, $X$, Jiang, D: Existence and uniqueness of positive solution for nonlinear singular 2 nth-order continuous and discrete Lidstone boundary value problems. Acta Math. Sci. Ser. B Engl. Ed. 831, 281-291 (2011)

28. Henderson, J, Luca, R: On a multi-point discrete boundary value problem. J. Differ. Equ. Appl. 19, 690-699 (2013)

29. Pielou, E: An Introduction to Mathematical Ecology. Wiley-Interscience, New York (1974)

30. Kuang, Y: Delay Differential Equations with Applications in Population Dynamics. Academic Press, New York (1993)

31. Gopalasamy, K: Stability and Oscillation in Delay Equation of Population Dynamics. Kluwer Academic, Dordrecht (1992)

32. Teng, Z: On the persistence and positive periodic solution for planar competing Lotka-Volterra systems. Ann. Differ. Equ. 13, 275-286 (1997)

33. Gaines, R, Mawhin, J: Coincidence Degree and Nonlinear Differential Equations. Springer, Berlin (1977)

34. Fang, M, Wang, K: Periodic solutions of a discrete time nonautonomous ratio-dependent predator-prey system. Math. Comput. Model. 35, 951-961 (2002)

35. Li, W, Huo, H: Positive periodic solutions of delay difference equations and applications in population dynamics. J. Comput. Appl. Math. 176, 357-369 (2005) 